\title{
Exact Algorithms for Biclique Coloring
}

\author{
Guilherme C. M. Gomes, ${ }^{(1)}$ Carlos V. G. C. Lima, \\ Vinicius F. dos Santos
}

\begin{abstract}
A $k$-biclique-coloring of $G$ is $k$-coloring of the vertices of $G$ with no maximal monochromatic biclique. A recent work showed that determining if such a coloring exists is $\Sigma_{2}^{\mathrm{P}}$-complete for $k>2$. We present algorithms to determine if a graph has a $k$-biclique-coloring: an $\mathcal{O}^{*}\left(4^{n}\right)$-time algorithm using exact cover, and an $\mathcal{O}^{*}\left((k+2)^{2 d}\right)$ time FPT algorithm, where $d$ is the neighborhood diversity of $G$.
\end{abstract}

\section{Introduction}

Coloring problems are among the most studied ones in graph theory. The VERTEX COLORING problem asks if it is possible to assign one of $k$ possible colors for each vertex of a graph in such a way that adjacent vertices have different colors or, equivalently, in such a way that no edge is monochromatic. Variants and generalizations of this problem have been proposed. Among them, the Clique Coloring problem asks whether or not we can $k$-color the vertices of a graph such that no maximal clique is monochromatic, and was shown to be $\Sigma_{2}^{\mathrm{P}}$-Complete [9] even we fix $k=$ 2, but solvable in $\mathcal{O}^{*}\left(4^{n}\right)$-time [3]. On the other hand, the Biclique

2020 AMS Subject Classification: 05C15, $05 \mathrm{C} 85$.

Keywords and Phrases: Biclique coloring · FPT algorithms · Exact algorithms. This research was partially supported by Capes, CNPq and FAPEMIG. 
COLORING problem asks for a $k$-coloring of the input graph such that no maximal biclique is monochromatic. Similarly to Clique Coloring, BiClique Coloring was shown to be a $\Sigma_{2}^{\mathrm{P}}$-Complete problem in [5], even if the number of colors is fixed to 2, while in [7] it is shown checking if a coloring is a biclique coloring is coNP-Complete. Little is known about the complexity of Biclique Coloring on graph classes. For unichord-free graphs, a polynomial-time algorithm for BICLIQUE COLORING is given by Macêdo Filho et al. [8]. They also show that the smallest number of colors needed to biclique-color unichord-free graphs is either equal to or one unit greater than the size of the largest true twin class. In [7], a polynomial-time algorithm for powers of cycles and powers of paths is presented. Finally, Groshaus et al. [5] deal with $H$-free graphs, for every $H$ on three vertices, with BICLIQUe COLORING being polynomial for $H \in\left\{K_{3}, P_{3}, \overline{P_{3}}\right\}$ and NP-Complete for $\overline{K_{3}}$-free graphs; moreover they show that the problem is NP-Complete for diamond-free graphs and split graphs, and polynomial for threshold graphs. Both Clique Coloring and Biclique Coloring can be seen as hypergraph coloring problems [1] where we want to color its vertices such that no hyperedge is monochromatic. In this paper we present the first exact algorithms for BICLIQUe Coloring, beginning with a $\mathcal{O}^{*}\left(4^{n}\right)$-time algorithm, then show a $\mathcal{O}^{*}\left((k+2)^{2 d}\right)$-time FPT algorithm parameterized by the neighborhood diversity $d$ and the number of colors. We conclude with directions for future work.

\section{Preliminaries}

We denote by $[n]$ the set $\{1, \ldots, n\}$ and by $2^{S}$ the powerset of a set $S$. Given a set $V$ and a subset $S \subseteq V$, let us denote by $\bar{S}=V \backslash S$. An algorithm is FPT if, given a parameter $k$, its running time is bounded by $f(k) n^{\mathcal{O}(1)}$, for some computable function $f$. A $k$-coloring of a graph $G$ is a function $\varphi: V(G) \mapsto[k]$; the coloring of a set $X \subseteq V(G)$ is defined as $\varphi(X)=\bigcup_{v \in X}\{\varphi(v)\}$ and $X$ is monochromatic if $|\varphi(X)|=1$. A $k$-coloring is a proper $k$-biclique-coloring if and only if no (inclusion- 
wise) maximal induced biclique of $G$ is monochromatic. The Biclique Coloring problem is the problem of deciding, given $G$ and $k$, whether or not $G$ has a proper $k$-biclique-coloring. Unless noted, all colorings are "proper". Two vertices $u, v \in V(G)$ are false twins if and only if $N(u)=N(v)$; they are true twins if and only if $N[u]=N[v]$. A type is a maximal subset $D \subseteq V(G)$ of true twins or false twins. Being of the same type is an equivalence relation and partitioning $G$ into the equivalence classes can be done in polynomial time [4]. The neighborhood diversity of $G$ is the number of types of $G$.

A hypergraph $\mathcal{H}=(V(\mathcal{H}), \mathcal{E})$ has $V(\mathcal{H})$ as its vertex set and $\mathcal{E} \subseteq 2^{V}$ as its hyperedge set. A graph $G$ is said to be a host of $\mathcal{H}$ if $V(G)=V(\mathcal{H})$, every hyperedge of $\mathcal{H}$ induces a connected subgraph of $G$ and every edge of $G$ is contained in at least one hyperedge of $\mathcal{H}$. For a hypergraph $\mathcal{H}$, a transversal is a set $X \subseteq V(\mathcal{H})$ that, for every hyperedge $\epsilon \in \mathcal{E}(\mathcal{H})$, $X \cap \epsilon \neq \emptyset$. If $X$ is not a transversal we say that it is an oblique. The biclique hypergraph $\mathcal{H}_{\mathcal{B}}(G)$ of a graph $G$ is the hypergraph on the same vertex set of $G$ and with hyperedge set equal to the family of maximal bicliques of $G$, denoted by $\mathcal{B}(G)$. We denote by $\mathbb{T}_{\mathcal{B}}(G)$ the family of all transversals of the biclique hypergraph of $G$, by $\mathbb{T}_{B}^{*}(G)$ the family of complements of transversals, and by $\mathbb{O}_{B}(G)$ the family of all obliques $\mathcal{B}(G)$.

We also make use of the following results and problems studied in the literature; we use Lemma 2.1 and Theorem 2.1 to show an $\mathcal{O}^{*}\left(4^{n}\right)$-time algorithm for Biclique Coloring, and employ Theorem 2.2 to solve Biclique Coloring parameterized by neighborhood diversity and the size of the largest true twin class in $\mathcal{O}^{*}\left((b+2)^{2 d}\right)$-time.

\section{EXACT COVER}

Instance: A set $A=\left\{a_{1}, \ldots, a_{n}\right\}$, a covering family $\mathcal{F} \subseteq 2^{A}$ and $k \in \mathbb{N}$. Question: Is it possible to $k$-partition $A$ into $\varphi$ such that $\varphi \subseteq \mathcal{F}$ ?

Set Multicover

Instance: A set $A=\left\{a_{1}, \ldots, a_{n}\right\}$, a family $\mathcal{F} \subseteq 2^{A}, k \in \mathbb{N}$ and $c: A \mapsto \mathbb{N}$. Question: Can we $k$-cover $A$ with $\varphi \subseteq \mathcal{F}$ so that $\left|\left\{\varphi_{i} \mid a_{j} \in \varphi_{i}\right\}\right| \geq c\left(a_{j}\right)$ for every $a_{j} \in A$ ? 
Lemma 2.1 ([3]). The down closure $\mathcal{F}_{\downarrow}=\{X \subseteq V \mid \exists Y \in \mathcal{F}, X \subseteq Y\}$ of a family $\mathcal{F} \subseteq 2^{V}$ can be enumerated in $\mathcal{O}^{*}\left(\left|\mathcal{F}_{\downarrow}\right|\right)$-time.

Theorem 2.1 ([2]). EXACT COVER is solvable in $\mathcal{O}^{*}\left(2^{n}|\mathcal{F}|\right)$-time.

Theorem $2.2([6])$. Set Multicover can be solved in time $\mathcal{O}^{*}\left((b+2)^{n}|\mathcal{F}|\right)$, with $b$ the maximum coverage requirement.

\section{Exact exponential algorithm}

Following [3], we first show the relationships between structures of $\mathcal{B}(G)$ and colorings, then use these to build an $\mathcal{O}^{*}\left(4^{n}\right)$-time algorithm for BICLIQUe Coloring by stating it as an EXACT Cover instance.

Lemma 3.1. A k-partition $\varphi=\left\{\varphi_{1}, \ldots, \varphi_{k}\right\}$ is a $k$-biclique-coloring of $G$ if and only if for every $i, \overline{\varphi_{i}} \in \mathbb{T}_{B}(G)$.

Proof. For the forward direction, suppose that there exists some $\varphi_{i}$ such that $\overline{\varphi_{i}} \notin \mathbb{T}_{B}(G)$. This implies that there exists some $B \in \mathcal{B}(G)$ such that $B \cap \overline{\varphi_{i}}=\emptyset$ and that $B \subseteq \varphi_{i}$; that is, $|\varphi(B)|=1$, which is a contradiction, since $\varphi$ is a $k$-biclique-coloring. For the converse, let $\varphi$ be a $k$-partition of $G$ with $\overline{\varphi_{i}} \in \mathbb{T}_{B}(G)$, but suppose that $\varphi_{k}$ is not a $k$-biclique-coloring. That is, there exists some maximal biclique $B \in \mathcal{B}(G)$ such that $B \subseteq \varphi_{i}$ for some $i$, which implies $B \cap \overline{\varphi_{i}}=\emptyset$ and $\overline{\varphi_{i}} \notin \mathbb{T}_{B}(G)$, a contradiction.

Lemma 3.2. The maximal obliques of $\mathcal{H}_{B}(G)$ are exactly the complements of the maximal bicliques of $G$.

Proof. Let $X \in \mathbb{O}_{B}$ be a maximal oblique. By definition, there exists some $B \in \mathcal{B}(G)$ such that $X \cap B=\emptyset$, which implies that $X \subseteq \bar{B}$. Note that, if $X \subset \bar{B}$, there is some $v \in \bar{B} \backslash X$, which implies that $(X \cup\{v\}) \cap B=$ $\emptyset$ and that $X$ is not a maximal oblique. Let $B \in \mathcal{B}(G)$. By definition, $\bar{B} \in \mathbb{O}_{B}$ and must be maximal because $\{B, \bar{B}\}$ is a partition of $V(G)$.

Corollary 3.1. Given a graph $G$ and a subset $X \subseteq V(G)$, there exists an $\mathcal{O}\left(n^{2}\right)$-time algorithm to determine if $X$ is a maximal oblique. 
Theorem 3.1. Biclique Coloring is solvable in $\mathcal{O}^{*}\left(4^{n}\right)$-time.

Proof. Our goal is to make use of Theorem 2.1 to solve an instance of EXACT COVER, with $A=V(G), \mathcal{F}=\mathbb{T}_{B}^{*}(G)$ and $k$ the partition size. Lemma 3.1 guarantees that there is a solution to our instance of BICLIQUE Coloring if and only if the corresponding EXACT COVER instance is solvable. To compute $\mathbb{T}_{B}^{*}(G)$, for each $X \in 2^{V(G)}$, we use Lemma 3.2 and Corollary 3.1 to say whether or not $X$ is a maximal oblique of $\mathcal{H}_{B}(G)$. Next, we compute $\mathbb{O}_{B}(G)$ from its maximal elements using Lemma 2.1, use the fact that $\mathbb{T}_{B}(G)=2^{V(G)} \backslash \mathbb{O}_{B}(G)$, and complement each transversal to obtain $\mathbb{T}_{B}^{*}(G)$. This procedure takes $\mathcal{O}^{*}\left(2^{n}\right)$-time to construct $\mathbb{T}_{B}^{*}(G)$ and $\mathcal{O}^{*}\left(4^{n}\right)$-time to obtain a coloring using Theorem 2.1 .

\section{$4 \quad$ FPT algorithm}

Suppose that we are already given a partition $\left\{D_{1}, \ldots, D_{d}\right\}$ of $V(G)$ in types. If $D_{i}$ is composed of true twins, we say that it is a true twin class and denote $D_{i}$ as $T_{i}$. Otherwise, $D_{i}$ is a false twin class denoted by $F_{i}$. Our algorithm builds an induced subgraph $H$ of $G$ that contains representatives of each true and false twin class. Afterwards, we build and solve an instance of SET Multicover derived from $H$, which we show to be equivalent to the original BiClique Coloring instance of $G$.

Observation 1. Given $G$ and a true twin class $T_{i}$ of $G$, any $k$-bicliquecoloring $\varphi$ of $G$ has $\left|\varphi\left(T_{i}\right)\right|=\left|T_{i}\right|$.

Lemma 4.1. If $F$ is a false twin class of $G$, any $k$-biclique-coloring $\varphi^{\prime}$ of $G$ can be changed into a $k$-biclique-coloring $\varphi$ where $|\varphi(F)| \leq 2$.

Proof. If $\left|\varphi^{\prime}(F)\right| \leq 2$, then $\varphi=\varphi^{\prime}$. Otherwise, there exist $f_{1}, f_{2}, f_{3} \in F$ with three different colors. Since every maximal biclique $B$ of $G$ that intercepts $F$ has $F \subset B$, we have $\left|\varphi^{\prime}(B)\right| \geq 3$. By making $\varphi\left(f_{1}\right)=\varphi^{\prime}\left(f_{1}\right)$ and $\varphi\left(f_{3}\right)=\varphi\left(f_{2}\right)=\varphi^{\prime}\left(f_{2}\right)$, we have $|\varphi(B)| \geq|\varphi(F)| \geq 2$. Repeating this process until $|\varphi(F)|=2$ does not make any biclique monochromatic. 

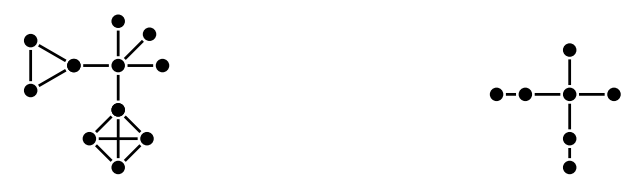

Figure 1: A graph and its projected graph

Definition 4.1 (Projection and Lifting). Let $T_{i}$ and $F_{j}$ be as previously defined; in the following $t_{i}^{\prime} \in T_{i}$ and $f^{\prime 1}{ }_{j}, f^{\prime 2}{ }_{j} \in F_{j}$. We define the following projection rules: $\forall v \in T_{i}, \operatorname{Proj}(v)=\left\{t_{i}^{\prime}\right\} ;$ for $f_{j}^{\prime 1} \in F_{j}, \operatorname{Proj}\left(f_{j}^{\prime 1}\right)=\left\{f_{j}^{\prime 1}\right\}$; $\forall u \in F_{j} \backslash\left\{f_{j}^{1}\right\}, \operatorname{Proj}(u)=\left\{f_{j}^{\prime 2}\right\}$ and $\operatorname{Proj}(X)=\bigcup_{u \in X} \operatorname{Proj}(u)$. Lifting rules are defined as $\operatorname{Lift}\left(t_{i}^{\prime}\right)=\left\{t_{i}\right\} ; \operatorname{Lift}\left(f_{j}^{\prime 1}\right)=\left\{f_{j}^{1}\right\} ; \operatorname{Lift}\left(f_{j}^{\prime 2}\right)=F_{j} \backslash\left\{f_{j}^{1}\right\}$ and $\operatorname{Lift}(Y)=\bigcup_{u \in Y} \operatorname{Lift}(u)$. Note that $\operatorname{Proj}(\operatorname{Lift}(X))=X$.

For the remainder of this section, $(G, k)$ is the input to Biclique ColORING and $H$ is the projected graph of $G$, i.e. $V(H)=\operatorname{Proj}(V(G))$ and $v_{i}^{\prime} v_{j}^{\prime} \in E(H)$ if and only if vertices in $D_{i}$ are adjacent to vertices in $D_{j}$. Our Set Multicover instance is the tuple $\left(V(H), \mathbb{T}_{B}^{*}(H), k, c\right)$ where $c\left(t_{i}^{\prime}\right)=\left|T_{i}\right|$ for each true twin class $T_{i}$ and $c\left(f_{j}^{\prime 1}\right)=c\left(f_{j}^{\prime 2}\right)=1$ for each false twin class $F_{j}$. Observation 2 follows from the fact that $\mathbb{T}_{B}^{*}(H)$ is closed under the subset operation.

Observation 2. If there is a minimum $k$-multicover $\psi$ of $V(H)$ by $\mathbb{T}_{B}^{*}(H)$, then there exists a minimum $k$-multicover $\psi^{\prime}=\left\{\psi_{1}, \ldots, \psi_{k}\right\}$ such that $\left|\left\{j \mid u \in \psi_{j}\right\}\right|=c(u)$ for every $u \in V(H)$.

Lemma 4.2. If $B^{\prime} \in \mathcal{B}(H)$, then $B=\operatorname{Lift}\left(B^{\prime}\right) \in \mathcal{B}(G)$. If $B \in \mathcal{B}(G)$ and $B$ is not contained in any true twin class, then $B^{\prime}=\operatorname{Proj}(B) \in \mathcal{B}(H)$.

Proof. For the first statement, note that $B$ is a biclique by the definition of Lift and the fact that $B^{\prime}$ is a biclique. By the contrapositive, suppose that $B \notin \mathcal{B}(G)$ and that $u \in V(G) \backslash B$ is such that $B \cup\{u\}$ is a (not necessarily maximal) biclique of $G$. Note that either: (i) if $u \in F_{j}$ then $F_{j} \nsubseteq B$ and $\operatorname{Proj}(u) \cap B^{\prime}=\emptyset$, otherwise we would have $\operatorname{Lift}(\operatorname{Proj}(u)) \subseteq B$, which is a contradiction since $u \in \operatorname{Lift}(\operatorname{Proj}(u))$; or (ii) if $u \in T_{i}$ then $T_{i} \cap B=\emptyset$, which implies that $\operatorname{Proj}(u) \nsubseteq B^{\prime}$. Since $B \cup\{u\}$ is a $\operatorname{biclique,~} \operatorname{Proj}(u)$ is 
adjacent to only one partition of $B^{\prime}$. Since $\operatorname{Proj}(u) \nsubseteq B^{\prime}, \operatorname{Proj}(B \cup\{u\})=$ $\operatorname{Proj}(B) \cup \operatorname{Proj}(u)=B^{\prime} \cup \operatorname{Proj}(u)$ is a biclique of $H$ and $B^{\prime}$ is not maximal.

For the second statement, by the definition of Proj, $B^{\prime}$ is a biclique of $H$ with parts $X, Y$. Suppose that $B^{\prime}$ is not maximal, i.e. there is $u^{\prime} \in V(H)$ such that $B^{\prime} \cup\left\{u^{\prime}\right\}$ is a biclique of $H$, and let $u \in \operatorname{Lift}\left(u^{\prime}\right)$. By the definition of Lift, it follows that $u$ is adjacent to only one part of $B$, say Lift $(X)$. Thus, for each $v \in \operatorname{Lift}(Y)$, uv $\notin E(G)$, otherwise there would be $v^{\prime} \in \operatorname{Proj}(v)$ with $u^{\prime} v^{\prime} \in E(H)$, implying that $B \cup\{u\}$ is a biclique of $G$.

Lemma 4.3. $X \subseteq V(H)$ is in $\mathbb{T}_{B}^{*}(H)$ if and only if $\operatorname{Lift}(X) \in \mathbb{T}_{B}^{*}(G)$.

Proof. Recall that $X \in \mathbb{T}_{B}^{*}(H)$ if and only if there is no $Y \in \mathcal{B}(H)$ with $Y \subseteq X$. Note that, for every $B^{\prime} \in \mathcal{B}(H), B^{\prime} \nsubseteq X$ implies that $\operatorname{Lift}\left(B^{\prime}\right) \nsubseteq$ $\operatorname{Lift}(X)$, since no two vertices of $H$ are lifted to the same vertex of $G$, and $\operatorname{Lift}\left(B^{\prime}\right) \in \mathcal{B}(G)$ due to Lemma 4.2. Moreover, no biclique of $G$ contained in a true twin class can be a subset of $\operatorname{Lift}(X)$. Thus, $\operatorname{Lift}(X)$ contains a maximal biclique $B$ only if $\operatorname{Proj}(B) \subseteq X$ and $\operatorname{Proj}(B) \notin \mathcal{B}(H)$, which is impossible due to Lemma 4.2 and the maximality of $B$. Taking the contrapositive, $X \notin \mathbb{T}_{B}^{*}(H)$ implies that there is some maximal biclique $B^{\prime}$ of $H$ such that $B^{\prime} \subseteq X$. This implies that $\operatorname{Lift}\left(B^{\prime}\right) \subseteq \operatorname{Lift}(X)$, and, since $\operatorname{Lift}\left(B^{\prime}\right) \in \mathcal{B}(G)$ due to Lemma 4.2 , it holds that $\operatorname{Lift}(X) \notin \mathbb{T}_{B}^{*}(G)$.

Theorem 4.1. $\psi$ is a k-multicover of $H$ if and only if $G$ is k-bicliquecolorable.

Proof. Recall that a $k$-partition is a $k$-biclique-coloring if and only if all elements of the partition belong to $\mathbb{T}_{B}^{*}(G)$. By the construction of our set multicover instance, we have that, for each $\psi_{i}, \psi_{i} \in \mathbb{T}_{B}^{*}(H)$. By making $\varphi=\left\{\operatorname{Lift}\left(\psi_{1}\right), \ldots, \operatorname{Lift}\left(\psi_{k}\right)\right\}$, and recalling Observation 2, we have that each vertex $u \in V(H)$ is covered exactly $c(u)$ times; moreover, since true twins appear multiple times and types are equivalence relations, we can attribute to each $v \in T_{i}$ any of the $\left|T_{i}\right|$ colors available, as long as no two receive the same color. That is, if $T_{i}=\left\{v_{1}, \ldots, v_{\ell}\right\}$ and $t_{i}^{\prime}$ is covered by $\left\{\psi_{i_{1}}, \ldots, \psi_{i_{\ell}}\right\}$, we assign to $v_{j}$ the color $i_{\ell}$. Therefore, $\varphi$ is a $k$-partition of 
$V(G)$ and, due to Lemma 4.3 , every $\operatorname{Lift}\left(\psi_{i}\right)$ is a complement of transversal, implying that $\varphi$ is a proper $k$-biclique-coloring of $G$. For the converse, we first make use of Lemma 4.1 to guarantee that every false twin class is in at most two color classes. In particular, if two colors are required we force $f_{j}^{1}$ to have the smallest color and $F_{j} \backslash\left\{f_{j}^{1}\right\}$ to have the other one. Afterwards, for every color class $\varphi_{i}$, we take $\psi_{i}=\operatorname{Proj}\left(\varphi_{i}\right)$. Note that, each color class has at most one element of each $T_{i}$ and, for each $F_{j}$ and any two distinct color classes $\varphi_{l}, \varphi_{r}, \operatorname{Proj}\left(\varphi_{l}\right) \cap \operatorname{Proj}\left(\varphi_{r}\right) \cap \operatorname{Proj}\left(F_{j}\right)=\emptyset$, since $f_{j}^{1}$ has a different color from $F_{j} \backslash\left\{f_{j}^{1}\right\}$. These observations guarantee that $\operatorname{Lift}\left(\psi_{i}\right)=\varphi_{i}$ and, because of Lemma $4.3, \psi_{i} \in \mathbb{T}_{B}^{*}(H)$. Finally, $\psi=\left\{\psi_{1}, \ldots, \psi_{k}\right\}$ is a valid $k$-multicover of $H$ since the vertices of $V(H)$ are sufficiently covered.

Note that the size of the largest true twin class is exactly the largest coverage requirement $b$ of our SeT Multicover instance. Moreover, since we need at least $b$ colors to biclique color $G$, it holds that $b \leq k$.

Theorem 4.2. Biclique Coloring can be solved in $\mathcal{O}^{*}\left((k+2)^{2 d}\right)$.

Another option would be not to contract true twin classes, keeping all such vertices in the projected graph, which would effectively yield a kernel linear on the product $k d$, and, even if we use Theorem 3.1, we would still take $\mathcal{O}^{*}\left(4^{k d}\right)$-time, which is no better than $(k+2)^{2 d} \approx 2^{2 d \log k}$.

\section{Conclusions and Future Work}

In this work we presented two exact algorithms for the BiCLIQue CoLORING problem. We first showed that, much like Clique Coloring, Biclique Coloring can be solved in $\mathcal{O}^{*}\left(4^{n}\right)$-time using the inclusionexclusion principle, and that the problem is solvable in FPT time when parameterized by the number of colors and neighborhood diversity of the input graph. While these are only initial results, further investigations into the parameterized complexity of the problem could be of interest. In particular, we leave the parameterization by neighborhood diversity as an 
open question, but are also interested in other structural parameterizations, such as treewidth and max leaf number. 


\section{References}

[1] Berge, C.: Hypergraphs: combinatorics of finite sets, vol. 45. Elsevier (1984)

[2] Björklund, A., Husfeldt, T., Koivisto, M.: Set partitioning via inclusion-exclusion. SIAM J. Comput. 39, 546-563 (2009)

[3] Cochefert, M., Kratsch, D.: Exact algorithms to clique-colour graphs. In: International Conference on Current Trends in Theory and Practice of Informatics. pp. 187-198. Springer (2014)

[4] Ganian, R.: Using neighborhood diversity to solve hard problems. arXiv preprint arXiv:1201.3091 (2012)

[5] Groshaus, M., Soulignac, F.J., Terlisky, P.: Star and biclique colouring and choosability. J. Graph Algorithms Appl. 18(3), 347-383 (2014)

[6] Hua, Q.S., Yu, D., Lau, F.C.M., Wang, Y.: Exact algorithms for set multicover and multiset multicover problems. In: Dong, Y., Du, D.Z., Ibarra, O. (eds.) Algorithms and Computation. ISAAC 2009. pp. 3444. Springer Berlin Heidelberg (2009)

[7] Macêdo Filho, H.B., Dantas, S., Machado, R.C., Figueiredo, C.M.: Biclique-colouring verification complexity and biclique-colouring power graphs. Discrete Appl. Math. 192, 65-76 (2015)

[8] Macêdo Filho, H.B., Machado, R.C., Figueiredo, C.M.: Cliquecolouring and biclique-colouring unichord-free graphs. In: LATIN'2016-Latin American Symposium on Theoretical Informatics. pp. 530-541. Springer (2012)

[9] Marx, D.: Complexity of clique coloring and related problems. Theor. Comput. Sci. 412(29), 3487-3500 (2011) 
Guilherme C. M. Gomes Carlos V. G. C. Lima

Universidade Federal de Minas Universidade Federal de Minas Gerais Gerais

Departamento de Ciência da Departamento de Ciência da Computação Computação

gcm.gomes@dcc.ufmg.br vinicius.lima@ufca.edu.br

Vinicius F. dos Santos

Universidade Federal de Minas

Gerais

Departamento de Ciência da Computação

viniciussantos@dcc.ufmg.br 